\title{
Modality-specific coding in matching letters
}

\author{
LARRY E. WOOD \\ University of Wisconsin-Parkside, Kenosha, Wisconsin 53140
}

\begin{abstract}
Auditory and visual similarity was manipulated in a same-different reaction-time task to investigate the use of modality-specific codes in same-different judgments for pairs of letters. Experiment 1 showed that letters presented simultaneously in the auditory and visual modalities were matched on the auditory dimension. In Experiment 2, the letters were presented sequentially, and the modality of the second letter was randomly varied. Subjects matched the pairs on the modality dimension of the second letter even though the modality could not be reliably predicted. In Experiment 3, subjects judged adjacent pairs of letters presented for $50 \mathrm{msec}$, and in one condition they also named the letters. Matches were made on visual codes in both conditions. In general, the results indicate that when subjects are instructed to determine if two letters are the same, the letters will be matched on a modality-specific code in a way that will minimize the information processing necessary to complete the match.
\end{abstract}

The present research investigated auditory and visual coding in recognition and classification of familiar stimuli such as letters of the alphabet. Because language symbols have a graphic (visual) representation and a spoken (auditory) representation, there is value in knowing how the two types of modality-specific codes are used in processing these stimuli. Research regarding this question has been conducted by Posner and his associates (Posner, 1969; Posner, Boies, Eichelman, \& Taylor, 1969; Posner \& Mitchell, 1967) and by others (Dainoff, 1970; Dainoff \& Haber, 1970). In these studies, subjects were presented with a pair of letters, either simultaneously or sequentially, and were instructed to respond as quickly as possible, indicating whether the letters were the same or different. Subjects responded "same" faster when two letters were both uppercase (physically same) than when one was presented in lowercase (name same). This result suggests that physical matches are made on visual information, but that name matches first require retrieval of the names (or auditory codes) from longterm memory and are then made on auditory information. The finding that visual similarity increases the latencies of physical matches (Clement \& Carpenter, 1970) and that auditory or acoustic similarity increases the latencies of name matches (Dainoff, 1970; Dainoff \& Habef, 1970) adds further to that conclusion.

Wood (1974) investigated modality-specific coding by inversely covarying the auditory and visual

The research was supported by a grant from the Wisconsin Alumni Research Foundation. The author wishes to express his gratitude to David Hignite and Kevin Petschow for their assistance in collecting and analyzing the data and to David F. Barone for his critical reading of an earlier version of this manuscript. Requests for reprints should be sent to Larry E. Wood, University of Wisconsin-Parkside, Kenosha, Wisconsin 53140. similarity of letter pairs rather than manipulating the case of the letters and the instructions for responding "same." The pairs were presented sequentially in the same or different modalities and at an interstimulus interval of 150 or $3,000 \mathrm{msec}$. The results indicated that the pairs were matched on a modality-specific code that corresponded to the modality of the second letter even when the two letters were presented in different modalities at the short interstimulus interval. The purpose of the present investigation was to extend the previous one and to study the effects of some additional factors on modality-specific coding in recognition of letters.

In order to study modality-specific coding, auditory and visual similarity between pairs of letters was inversely covaried as in the aforementioned study (Wood, 1974). Sets of letter pairs were selected so that one pair was high in auditory similarity but low in visual similarity (hi-aud/lo-vis), such as $\mathrm{ZC}$; and the other pair was low in auditory similarity but high in visual similarity (lo-aud/hi-vis), such as OC. Thus, there were sets of letter pairs in which one pair sounded similar but did not look similar and the other pair looked similar but did not sound similar. Comparison of the relative reaction times (RTs) of the two types of pairs enabled determination of which modality-specific code (if either) was being used for the comparisons. When auditory codes are used, hi-aud/lo-vis pairs should produce longer RTs than lo-aud/hi-vis pairs. When visual codes are used, the relative RTs to the two types of pairs should be in the reverse order. If neither dimension is used, then there should be no reliable similarity effects.

\section{EXPERIMENT 1}

As discussed earlier, the effects of auditory and visual similarity imply that letters are matched on 
modality-specific dimensions or codes. A further implication is that two letters must be coded in the same dimension in order for them to be compared on that dimension. Thus, when subjects are to compare letters that have been presented in different modalities or when the presentation and comparison modalities differ (visually presented letters with the same names), one or both of the letters need to be recoded before the match can be completed. The purpose of Experiment 1 was to study the importance of the letters being matched on a common, modalityspecific dimension as part of the comparison process. Posner $(1969,1973)$ has demonstrated that visual and auditory (name) codes of letters are independently stored in long-term memory. However, there is a highly overlearned equivalency relationship between the two types of codes that allow most literate people to move easily back and forth between them in a variety of ways. Because of the equivalency relationship, it would seem possible that one could decide if two letters were the same (equivalent) without actually matching the two stimuli or their representations. Rather, it is conceivable that the decision could be made logically by noting whether or not the sensory stimuli activated the same or equivalent codes in long-term memory.

Although similarity effects found in previous studies indicate that letters are compared by matching them on a common, modality-specific dimension, it is possible that particular characteristics of the tasks have biased subjects toward that type of processing. For example, presenting stimuli visually and then instructing subjects to respond same if letters are either physically the same or have the same names tends to emphasize modality-specific aspects of the task. In one study (Wood, 1974), the instructions did not emphasize modality-aspects of the task, but the stimuli were presented sequentially and the modality of the second stimulus remained constant throughout an experimental session. Thus, subjects were encouraged to adopt a strategy wherein the first stimulus was encoded or recoded in the modality of the second in preparation for a match as close to the sensory level as possible. Therefore, in the present study there was an attempt to avoid influencing subjects to compare letters by matching them on modality-specific codes. To accomplish this, pairs of letters were presented simultaneously in different modalities and subjects were simply asked to decide as quickly as possible whether they were the same or different. If subjects still matched the letters on modality-specific codes (as evidenced by similarity effects), it would seem to indicate that an actual match is a very important part of the comparison process.

In addition to the simultaneous condition, two control conditions were included in which the two letters of a pair were presented sequentially and in the same modality (both auditory or both visual). These conditions were intended to replicate earlier findings (Wood, 1974, Experiment I) that the similarity manipulations produce systematic differences in reaction time (RT) on the two types of pairs under different conditions. The previous results showed that, with auditory presentation of both letters, subjects made comparisons on that dimension, and similarly, visual presentation led to comparisons on the visual dimension.

\section{Method}

Subjects. The subjects were 10 students from introductory psychology courses serving as part of a course requirement. They were tested individually in three 50-min sessions on successive days.

Materials. The materials were the same as those used by Wood (1974) in which four sets of letter pairs were selected so that two pairs in each set contained the same second letter but different first letters appropriate to a hi-aud/lo-vis or lo-aud/hi-vis similarity relationship. The four sets were $Z C-O C, Z G-Q G$, VB-RB, and TP-RP. While four sets of pairs may appear to form an unnecessarily restrictive set of stimuli, it must be noted that they were the result of an attempt to construct letter pairs for which an opposite, dichotomous classification on both similarity dimensions could be made. Visual similarity was defined simply as the amount of overlap in area between two uppercase letters, and auditory similarity was defined in terms of common phonemes shared by the letters. Because auditory information is temporally dependent, it is necessary that differences in auditory similarity occur in the same position (i.e., on corresponding phonemes). Otherwise, any differences in RT as a function of similarity would be confounded with differences in the minimum latency required for detection of differences. This consideration plus the added constant of dichotomous extremes on the two similarity dimensions resulted in the testable stimulus set being restricted to four sets of two pairs. The assumption was made, of course, that results obtained using these pairs would not be restricted to the particular stimuli, but that these pairs would be maximally sensitive indicators of the processing of all letters. Each of the 8 test pairs was presented three times in a block of 60 trials. Because 6 of the 8 test pairs had a different first letter, 16 additional different pairs were constructed to create greater uncertainty regarding the second letter. Each of the initial letters was paired with $S$ and $K$ once, except for the initial letters $Z$ and $R$, which were used twice. The remaining 20 trials in each block of 60 were same pairs consisting of each initial letter being paired with itself three times, except for $\mathrm{ZZ}$ and $\mathrm{RR}$, which were used four times.

Apparatus. Visual stimuli were presented on an Industrial Electronics Engineers Binaview Display, and auditory stimuli were presented binaurally through headphones via a tape recorder. A BRS Electronics digital logic system was used to control the Binaview Display and to time responses. The output of the tape recorder was gated through the BRS system to synchronize auditory and visual stimuli and to control the interstimulus and intertrial intervals. Duration of the visual stimuli was $360 \mathrm{msec}$, the mean duration of the auditory stimuli as measured on an oscilloscope. The subjects responded by pushing one of two telegraph keys marked "same" or "different" and spaced $.348 \mathrm{~m}$ apart. The RTs were measured to the nearest millisecond.

Procedure. Each subject participated in all three conditions. Half of the subjects served in the auditory-auditory (A-A) condition the first day and the visual-visual (V-V) condition the second day; the other half served in the opposite order. All subjects received the auditory-visual (AV) condition on the third day. In each condition, subjects received three blocks of trials with each block consisting of six practice trials followed 
by the 60 stimulus pairs in random order. The members of each pair in the A-A and V-V conditions were separated by 1 sec from onset of the first stimulus to onset of the second and the pairs were separated by $3 \mathrm{sec}$. There was a 2 -min rest period between each block. The subjects were instructed to place the index finger of each hand on one of the keys and to try to make a correct response as quickly as possible on each trial. The same response was always assigned to the dominant hand since there was no interest in directly comparing same and different responses.

\section{Results and Discussion}

A median RT was computed from the correct responses for each pair, and the four medians for each similarity condition were averaged for each subject in each of the six similarity and modality combinations. The scores were then submitted to a two-way analysis of variance, which resulted in a reliable Modality by Similarity interaction, $F(2,27)$ $=23.90, \mathrm{p}<.001$. As shown in Table 1, the hi-aud/ lo-vis pairs yielded reliably longer RTs than the lo-aud/hi-vis pairs in the A-A condition, $F(1,18)=$ $43.29, \mathrm{p}<.001$, but in the $\mathrm{V}-\mathrm{V}$ condition, the hi-aud/lo-vis pairs produced reliably shorter RTs than did the lo-aud/hi-vis pairs, $F(1,18)=9.72$, $\mathrm{p}<.01$. Thus, matches were made with auditory codes in the A-A condition but with visual codes in the V-V condition. These results confirm earlier findings (Wood, 1974) that the similarity manipulations are differentially sensitive to other variables and are therefore useful for inferring modalityspecific coding.

In the AV condition, the hi-aud/lo-vis pairs led to reliably longer RTs than the lo-aud/hi-vis pairs, $F(1,18)=7.97, \mathrm{p}<.025$. This finding indicates that pairs were matched on the basis of the auditory codes, and hence that the visual member of the pair was recoded to its auditory equivalent prior to the match. Thus, even when the letters were presented simultaneously in different modalities, they were nevertheless matched on a modality-specific code. This finding seems to show that an actual match between the modality-specific codes of letters is an important step in the comparison process. Evidence for comparison by a matching process has also been found by Cooper and Shephard (1973). They presented subjects with letters in their normal versions or as mirror images and rotated from the upright position. These investigators concluded that the subjects performed the task by imagining the normal version of the letter rotated into the indicated orientation and by matching it against a permanently stored representation of the normal upright version of that character. It could be that a same-different judgment is based on a match because a child's learning to discriminate between various letters of the alphabet initially requires a systematic comparison of similarities and differences between them. In a similar vein, Posner (1970) found that subjects could discriminate letters from digits with-
Table 1

Mean Reaction Times, Standard Errors, and Error Rates (in percentages) as a Function of Presentation Modality, Similarity Condition, and Type of Response

\begin{tabular}{crrr}
\hline & \multicolumn{3}{c}{ Presentation Modality } \\
\cline { 2 - 4 } Similarity & A-A & V-A & AV \\
\hline & \multicolumn{3}{c}{ Different Responses } \\
High Auditory/Low Visual & 509 & 452 & 622 \\
Mean & 20 & 30 & 43 \\
SE & 2 & 2 & 2 \\
Error & & & \\
Low Auditory/High Visual & 442 & 484 & 593 \\
Mean & 20 & 24 & 36 \\
SE & 1 & 1 & 1 \\
Error & & Same & Responses \\
& 471 & 473 & 607 \\
Mean & 16 & 23 & 28 \\
SE & 5 & 5 & 9 \\
Error & & &
\end{tabular}

out using the names of the stimuli, but they did use letter names in discriminating vowels from consonants. Although it was not logically necessary in either case, Posner concluded that names were involved in the vowel-consonant discrimination because it is more likely learned in reference to the letter names $A, E, I, O, U$.

The recoding of the visual stimuli for matching in the AV condition is noteworthy because there seems to be nothing apparent in the structure of the task to cause it. One reason for matching on the auditory dimension could be that visual to auditory recoding is simply faster than auditory to visual recoding. Faster visual to auditory recoding may be possible because the entire letter is available simultaneously with visual presentation, whereas the sequential nature of auditory presentation may require a delay of $200-300 \mathrm{msec}$ (in present study) before all necessary aspects of the stimulus have been presented. Wood (1974) found that when the auditory member of an A-V pair followed the visual member by approximately $500 \mathrm{msec}$, the visual stimulus was recoded to its auditory equivalent for matching. Perhaps the delay caused by the sequential nature of auditory stimuli was enough to accomplish the same thing in the present study. Another possible reason for visual-to-auditory recoding is that most people have more practice with it than with auditoryto-visual recoding because they usually learn to name (aloud) letters before they learn to write them. In addition, most people continue to spend more time reading than writing during their normal daily activities. Finally, visual-to-auditory recoding has been found in immediate memory tasks (e.g., Conrad, 1964; Wickelgren, 1965), indicating a general propensity toward auditory coding unless an additional factor is present to cause visual coding to 
be more effective in the situation (Parks, Kroll, Salzberg, \& Parkinson, 1972).

A final result of some interest was the large increase in RT from the A-A and V-V conditions to the AV condition, even though the AV condition came after the subjects had had considerable practice at the basic task. The difference is most obvious from the same responses, which are shown at the bottom of Table 1 and is likely a result of two factors. The first is the need to recode the visual letter to its auditory equivalent in the AV task after both letters have been presented, causing an increase in RT. This conclusion agrees with one reached by Posner and Mitchell (1967) from results obtained in a cross-modality matching task using digits. The second factor contributing to the shorter latency in the A-A and V-V conditions is that, with sequential presentation, the matching process could potentially be completed at a much lower perceptual level. That is, output from processing the first letter could be used to prime lower level analyzers to detect its identity with the second. Facilitatory effects of advance information on matching latencies have been found by Beller (1971) and by Posner and Boies (1971) in tasks similar to the present one.

\section{EXPERIMENT 2}

The results of Experiment 1 indicate that stimuli were matched on a modality-specific dimension when an attempt was made to reduce sources of modalityspecific bias in the task by presenting stimuli simultaneously in different modalities. Even in this case, however, the dimension used for matching was the one which can potentially be viewed as the "second" one because of the sequential nature of auditory presentation. Additional evidence regarding the influence of the second stimulus on the matching dimension was obtained in a study by Tversky (1969). She asked subjects to match pairs of stimuli (names and faces) that had been associated in such a way that similar faces had dissimilar names and dissimilar faces had similar names (i.e., visual and "verbal" similarity were inversely covaried across the stimulus set). The pairs were presented sequentially, and the mode of the second stimulus was randomly varied on each trial so that one mode was used on $79 \%$ of the trials and the other mode was used on the remaining $21 \%$ of the trials. Tversky found that matching latencies varied as a function of the similarity of the second stimulus independently of both the mode of the first stimulus and the mode frequency of the second. Hence, the first stimulus was apparently recoded to the mode of the second for matching, even when the second was presented in an unexpected mode.

Unfortunately, Tversky (1969) always presented the second stimulus for a constant duration of $4 \mathrm{sec}$ regardless of the subjects' response latencies. This may account for matching in the mode of the second, unexpected stimulus and thus weakens her results in that regard.

Therefore, Experiment 2 was designed to investigate the influence of the second stimulus on the matching task when the modality of the second stimulus was uncertain. Specifically, pairs of letters were presented sequentially, with the modality of the first always being auditory. The modality of the second letter was randomly varied so that half the second letters were presented in each modality. Furthermore, a relatively short stimulus duration was used. If the modality of the second stimulus is an important factor in the matching process, then matches should be made in that modality even though the subject cannot reliably predict it on a given trial. On the other hand, if the modality of the second stimulus per se is not so important, then perhaps all matches would be made on the auditory dimension given that the first letter was always auditory and that there seems to be a general tendency toward matching on the auditory dimension as found in Experiment 1. Auditory and visual similarity were manipulated again in order to infer the dimensions on which matches were made.

\section{Method}

Subjects. The subjects were 30 introductory psychology students serving as part of a course requirement. They were tested individually in a single 50 -min session.

Materials and Apparatus. The materials and apparatus used were the same as in Experiment 1.

Procedure. The procedure was the same as in Experiment 1, with the following exceptions. Fifteen subjects served in each of two conditions, which differed only in the modality of the second letter of each test pair (see Materials section of Experiment 1). In the auditory-auditory (A-A) condition the second letter of each test pair was always presented aurally, and in the auditory-visual (A-V) condition the second letter of each test pair was always presented visually. The second letter of the remaining (nontest) different pairs and all of the same pairs was distributed randomly between the auditory and visual modalities so that half of all second letters in both conditions were presented in each modality.

\section{Results and Discussion}

A mean RT for each subject for the two similarity conditions (hi-aud/lo-vis and lo-aud/hi-vis) was calculated as in Experiment 1. A two-way analysis of variance performed on these scores yielded a reliable Similarity by Modality (of the second letter) interaction, $F(1,28)=20.36, p<.001$. As shown in Table 2, the hi-aud/lo-vis pairs led to longer RTs than the lo-aud/hi-vis pairs, $F(1,28)=9.42, \mathrm{p}<.01$, when the second letter was auditory. In contrast, the hi-aud/lo-vis pairs produced shorter RTs than the lo-aud/hi-vis pairs, $\mathrm{F}(1,28)=10.63$, $\mathrm{p}<.01$, when the second letter was visual. These results indicate that the letters were matched on the modality dimen- 
Table 2

Mean Reaction Times, Standard Errors, and Error Rates (in percentages) as a Function of Presentation Modality and Similarity Condition

\begin{tabular}{crr} 
& \multicolumn{2}{c}{ Presentation Modality } \\
\cline { 2 - 3 } Similarity Condition & A-A & A-V \\
\hline High Auditory/Low Visual & \multicolumn{2}{c}{ Different Responses } \\
Mean & 568 & 559 \\
SE & 22 & 14 \\
Error & 1 & 2 \\
Low Auditory/High Visual & & \\
Mean & 552 & 576 \\
SE & 25 & 16 \\
Error & 3 & 1 \\
& \multicolumn{2}{c}{ Same Responses } \\
Mean & 587 & 596 \\
SE & 15 & 17 \\
Error & 4 & 2 \\
\hline
\end{tabular}

sion of the second letter even though the first letter was always auditory.

The matching of letter pairs in the modality dimension of the second letter is noteworthy because the modality could not be predicted by the subjects. The results agree with those of Tversky (1969) and suggest that the decision to match stimuli on a particular dimension is not made until the second stimulus is presented. The fact that the RTs are longer than those in the sequential conditions of Experiment 1 would also be expected if the decision for the matching dimension were delayed until presentation of the second letter.

The simplest explanation for the results would be that subjects recoded the first stimulus to the visual modality on the A-V trials as soon as they became aware that the second letter was visual. This strategy would agree with the results and conclusions from memory search tasks when the memory set and target are presented in different modes (Chase \& Calfee, 1969; Cruse \& Clifton, 1973; Swanson, Johnson, \& Briggs, 1972). In these studies, each item of the memory set appears to be matched in the mode of the target. Hence, investigators have concluded that each memory item is recoded to the mode in which the target is presented prior to each match. There is a difficulty with this interpretation for the present study, however. Because recoding would need to take place only in the A-V condition, the RTs for those trials should be longer than those for the A-A condition. However, neither the combined mean RTs for the different responses nor the same responses (see Table 2) were reliably different across the two conditions ( $\mathrm{F}<1$ in both cases).

A better interpretation of the present findings is one proposed by Clifton, Cruse, and Gutschera (1973) to explain results obtained in a memory search task wherein memory and target items were different, but equivalent, representations. These authors concluded that subjects recoded memory items at the time of presentation and stored both representations in memory simultaneously. The targets were compared to the memory set that matched the type of representation of the target. In the present experiment, such a strategy would be useful because the recoding could take place at the time of the presentation of the first letter followed by matching on the appropriate modality as soon as the second letter was encoded. There would be no reason to expect the overall RTs to differ between the two conditions, because matching would not have to await the recoding process on the A-V trials. However, the uncertainty associated with the second letter and the need to maintain both codes could still cause the RTs to be longer than in the A-A and V-V conditions of Experiment 1.

\section{EXPERIMENT 3}

Perhaps one reason the modality of the second stimulus so strongly influences the matching process (as shown in Experiment 2) is because matching in that dimension guarantees minimal processing of at least one of the stimuli. Because the first stimulus is already being held in memory, perhaps less processing capacity is required to recode it to the other modality and maintain both codes than to hold the first stimulus in memory while also extensively processing the second one. In an attempt to study this possibility further, Experiment 3 was conducted to see if the matching process would be affected when subjects were (1) prevented from directly using the physical stimuli as a basis for matching and (2) when they were required to recode both stimuli even though the recoding was not required for the matching part of the task. In the first case, a pair of letters was presented visually for a duration short enough to prevent multiple fixations $(50 \mathrm{msec})$. Under these circumstances, a match would have to be made from information in the short-term visual store (cf. Sperling, 1960 ) at the minimum. In the second case, the stimuli were presented for only $50 \mathrm{msec}$ and the subjects were also required to name them following the match. The naming response would require the subjects to recode the visual stimuli to their auditory equivalent for output. Hershenson and Price (1973) concluded that the names of the stimuli were implicated in the match in both of these situations. However, their data were inconclusive on this point. Furthermore, the findings of Experiment 2 would seem to argue that the match would be done with a minimal processing of the stimuli and would therefore be carried out on the visual codes.

In addition to the two $50-\mathrm{msec}$ conditions described previously, a control condition was in- 
cluded in which the letter pairs were shown for a duration of $500 \mathrm{msec}$, and the subjects were merely required to determine if they were the same or different. In accord with previous findings (Clement \& Carpenter, 1970), it was expected that comparisons in the control condition would be based on visual information. As in Experiments 1 and 2, auditory and visual similarity was manipulated as a means of determining the dimension on which the letters matched.

\section{Method}

Subjects. The subjects were 45 introductory psychology students tested individually in sessions lasting approximately $50 \mathrm{~min}$. They were randomly assigned to the three conditions as they arrived at the laboratory, with the restriction that the number in each condition never differed by more than one.

Materials. The materials were the same as those used as in Experiments 1 and 2.

Procedure. The stimuli were presented via a Gerbrands Model GB-3, three-field tachistoscope. Each subject received 24 practice trials followed by three blocks of 60 trials with a 2-min rest period between each block. The intertrial interval varied as a function of the time required to change cards in the tachistoscope. A trial consisted of the experimenter's saying "ready," followed by a fixation point for $1 \mathrm{sec}$, followed by a pair of uppercase letters (one on each side of the fixation point) shown for 50 or 500 msec. Each pair subtended a visual angle of approximately $1^{\circ}$ horizontally and $.5^{\circ}$ vertically. The subjects responded by saying either "same" or "different" into a microphone connected to a voice-operated relay. The RTs were measured to the nearest millisecond by a digital Klockounter, which was started simultaneously with the onset of the stimuli and was stopped by a closure of the voice-operated relay. The subjects were instructed to try to make a correct response as quickly as possible on each trial, and those in one of the $50-\mathrm{msec}$ conditions ( $50 \mathrm{msec}$ + name) were instructed to name the two letters following their same-different judgment.

\section{Results and Discussion}

For each subject, a median RT was calculated from correct responses to each of the eight test pairs (see Materials, Experiment 1). The four medians in each similarity condition were then used to calculate means for each subject. These scores were then submitted to a two-way analysis of variance, which resulted in a reliable main effect of Similarity, $F(1,42)=106.51, p<.001$. As shown in Table 3 , the hi-aud/lo-vis pairs led to reliably shorter RTs than the lo-aud/hi-vis pairs in all three of the duration and name conditions. This finding indicates that visual codes were used for all comparisons. Thus, apparently neither the short duration nor the need to name the stimuli following the same-different decision influenced subjects to use the auditory or name codes as the basis for comparison. This result strengthens the conclusions from Experiments 1 and 2 that (1) an actual matching of the stimuli at some level is part of the comparison process and (2) the match is carried out in a way that will minimize processing of the stimuli. The latter result is particularly interesting in light of the fact that both
Table 3

Mean Reaction Times, Standard Errors, and Error Rates (in percentages) as a Function of Similarity, Presentation Condition, and Response Type

\begin{tabular}{|c|c|c|c|}
\hline \multirow[b]{2}{*}{ Similarity } & \multicolumn{3}{|c|}{ Presentation Condition } \\
\hline & $500 \mathrm{msec}$ & $50 \mathrm{msec}$ & $\begin{array}{l}50 \mathrm{msec} \\
+ \text { names }\end{array}$ \\
\hline & \multicolumn{3}{|c|}{ Different Responses } \\
\hline $\begin{array}{l}\text { High Auditory/Low Visual } \\
\text { Mean } \\
\text { SE } \\
\text { Error }\end{array}$ & $\begin{array}{r}491 \\
11 \\
7\end{array}$ & $\begin{array}{r}587 \\
15 \\
5\end{array}$ & $\begin{array}{r}594 \\
17 \\
3\end{array}$ \\
\hline $\begin{array}{l}\text { Low Auditory/High Visual } \\
\text { Mean } \\
\text { SE } \\
\text { Error }\end{array}$ & $\begin{array}{r}549 \\
10 \\
6\end{array}$ & $\begin{array}{r}621 \\
12 \\
5\end{array}$ & $\begin{array}{r}636 \\
14 \\
3\end{array}$ \\
\hline Error & \multicolumn{3}{|c|}{ Same Responses } \\
\hline $\begin{array}{l}\text { Mean } \\
\text { SE } \\
\text { Error }\end{array}$ & $\begin{array}{r}572 \\
12 \\
10\end{array}$ & $\begin{array}{r}639 \\
13 \\
4\end{array}$ & $\begin{array}{r}690 \\
18 \\
4\end{array}$ \\
\hline
\end{tabular}

stimuli had to be recoded in the 50-msec + name condition for the naming operation.

The analysis of variance also showed a reliable main effect among the three duration and name conditions, $F(2,42)=7.92, p<.001$. There are two aspects of this result which are interesting. First, there was not a reliable difference between the two 50 -msec conditions, $F<1$, which suggests that the need to name the stimuli did not appreciably affect the absolute RT. This result implies that the retrieval of the name codes was performed independently of the matching process. It should be noted, however, that an analysis of same responses (also listed in Table 3) did show that RT was reliably longer, $F(1,42)=6.14, p<.025$, when the subjects were required to name the stimuli. This result is rather unexpected, because it would seem easier to use the same auditory code twice than to retrieve two different codes. The second interesting result from the duration and name conditions (reflected in both the same and different responses) was that RTs in the 500-msec condition were reliably faster than in the two 50-msec conditions, $F(1,42)=7.73$, p $<.01$. This finding may simply reflect some difficulty in recognizing a brief stimulus. As shown in Table 3, however, the error rates were actually smaller on an absolute basis for the 50-msec conditions. Perhaps an opportunity to successively scan the physical stimuli increases the speed of the match because processing capacity does not have to be used to maintain a rapidly fading sensory image as would be the case in the 50 -msec condition.

\section{SUMMARY}

The present study was an attempt to investigate some of the factors that influence modality-specific 
coding in recognition of letters. Experiment 1 demonstrated that when the task requires matching of two letters presented simultaneously in different modalities, the visual member will be recoded to its auditory equivalent for matching. Thus, matching the letters on modality-specific codes seems to be an important step in the comparison process even when it would not seem logically necessary. Experiment 2 showed that when the letters are presented sequentially, the matching dimension is determined by the modality of the second stimulus of a pair even when the modality is unpredictable. Finally, the results of Experiment 3 show that when a pair of letters is shown for a duration too short to allow multiple fixations, the match will be made on the visual dimension even though both letters are subsequently recoded to their auditory equivalents for naming. Thus, even though an actual match is performed as part of the comparison process, it appears to be carried out in a way that will minimize processing of the stimuli.

\section{REFERENCES}

BeLLER, H. K. Priming: Effects of advance information on matching. Joumal of Experimental Psychology, 1971, 87, 176-182.

Cense, W. G., \& Calfee, R. C. Modality and similarity effects in short-term recognition memory. Journal of Experimental Psychology, 1969, 81, 510-514.

Cemarert, D. E., \& CARPEnTER, J. S. Relative discriminability of visually presented letter pairs using a same-different choice reaction-time task. Psychonomic Science, 1970, 20, 363-365.

Cunfton, C., Jr., Cruse, D., \& Gutschera, K. D. Recoding processes in recognition: Some effects of presentation rate. Memory \& Cognition, 1973, 1, 387-394.

Conrad, R. Acoustic confusions in immediate memory. British Journal of Psychology, 1964, 55, 75-84.

COOPER, L. A., \& Shephard, R. N. The time required to prepare for a rotated stimulus. Memory \& Cognition, 1973, 1, 246-250.
CRUse, D., \& Cumton, C., JR. Recoding strategies and the retrieval of information from memory. Cognitive Psychology, 1973, 4, 157-193.

Datroff, M. J. Time course of visual and auditory encoding. Journal of Experimental Psychology, 1970, 86, 214-224.

Danoff, M. J., \& HaBer, R. N. Effect of acoustic confusability on levels of information processing. Canadian Journal of Psychology, 1970, 24, 98-108.

Herstenson, M., \& Price, K. P. Analysis of "physical" matching task for simultaneously presented pairs of letters. Journal of Experimental Psychology, 1973, 97, 198-203.

Parks, T. E., Kroll, N. E. A., Salzberg, P. M., \& Parkinson, S. R. Persistence of visual memory as indicated by decision time in a matching task. Journal of Experimental Psychology, $1972,92,437-438$

Posner, M. I. Abstraction and the process of recognition. In G. H. Bower \& J. T. Spence (Eds.), The psychology of learning and motivation (Vol. 3). New York: Academic Press, 1969.

PosNer, M. I. On the relationship between letter names and superordinate categories. Quarterly Journal of Experimental Psychology, 1970, 22, 279-287.

Posner, M. I. Cognition: An introduction. Glenview, Ill: Scott, Foresman, 1973.

Posner, M. I., Boies, S. J., Eichelman, W. H., \& Tayzor, R. L. Retention of visual and name codes of single letters. Joumal of Experimental Psychalogy Monograph, 1969, 79 (1, Pt. 2).

Posmer, M. I., \& BoIzs, S. J. Components of attention. Psychological Review, 1971, 74, 391-408.

Posner, M. I., \& Mrtchell, R. F. Chronometric analysis of classification. Psychological Review, 1967, 74, 392-409.

SPERLING, G. The information available in brief visual presentations. Psychological Monographs, 1960, 74(11, Whole No. 498).

Swanson, J. M., Johnson, A. M., \& Briggs, G. E. Recoding in a memory search task. Journal of Experimental Psychology. 1972, 93, 1-9.

TVERSKY, B. Pictorial and verbal encoding in a short-term memory task. Perception \& Psychophysics, 1969, 6, 225-233.

Wickelgren, W. A. Acoustic similarity and intrusion errors in short-term memory. Joumal of Experimental Psychology, 1965, 70, 102-108.

WooD, L. E. Visual and auditory coding in a memory matching task. Journal of Experimental Psychology, 1974, 102, 106-113.

(Received for publication August 11, 1976; revision received December $17,1976$. 